\title{
Phlebologische Sprechstunde für Einsteiger: Sklerosierungstherapie von Besenreisern
}

\section{A.L. Recke; B. Kahle}

Klinik für Dermatologie, Venerologie und Allergologie, Universitätsklinikum Schleswig-Holstein, Campus Lübeck

Für die Behandlung von kleinkalibrigen Varizen (retikuläre Varizen, Besenreiser) gilt die Sklerosierungsbehandlung als Methode der ersten Wahl.

Bei sachgerechter Durchführung ist die Verödungsbehandlung eine effiziente und nebenwirkungsarme Therapieform. Dennoch gibt es unmittelbare und im Intervall auftretende mögliche Nebenwirkungen wie allergische Reaktionen, Hautnekrosen, überschießende Sklerosierungsreaktion und Thrombophlebitis, Pigmentierungen und Matting sowie seltenere Nebenwirkungen, über die der Patient im Vorfeld aufgeklärt werden sollte. Nähere Informationen finden sich in der Leitlinie zur Sklerosierungsbehandlung der Varikose (1).

Ebenso finden sich in der Leitlinie (1) und der Fachinformation des verwendeten
Sklerosierungsmittels Polidocanol (Aethoxysklerol ${ }^{\circ}$ ) genaue Angaben zur Konzentration und zum Volumen, das maximal pro Injektion verabreicht werden sollte.

\section{Interessenkonflikt}

Nach Angaben der Autoren bestehen keine Interessenkonflikte.

\section{Ethische Richtlinien}

Für das Manuskript wurden keine Studien an Menschen oder Tieren durchgeführt.

\section{Literatur}

1. Rabe E, Gerlach H, Breu FX, Guggenbichler S, Stücker M, Pannier F. Leitlinie: Sklerosierungsbehandlung der Varikose der Deutschen Gesellschaft für Phlebologie. AWMF-Leitlinien-Register-Nr. 037/015, Entwicklungsstufe S1. Phlebologie 2012; 41(4): 206-221

2. Weiss RA, Sadock NS, Goldman MP, Weiss MA Post-sclerotherapy compression: controlled comparative study of duration of compression and its effects on clinical outcome. Dermatol Surg 1999; 25(2): 105-108.

3. Kern P, Ramelet AA, Wintschert R, Hayon D. Compression after sclerotherapy for teleangiectasias and reticular leg veins: A randomized controlled trial. J Vasc Surg 2007; 45: 1212-1215.

4. Nootheti PK, Cadag KM, Magpantay A, Goldman MP. Efficacy of graduated compression stockings for an additional 3 weeks after sclerotherapy treatment of reticular and telangiectatic leg veins. Dermatolog Surg 2009; 35: 53-57.

5. Noppeney T, Noppeney J, Cucuruz B. Kompression nach Sklerotherapie mit flüssigen und aufgeschäumten Agenzien. Phlebologie 2018; 47: 21-23.
Korrespondenzadresse

Dr. med. Anna Lena Recke

Klinik für Dermatologie, Venerologie und Allergologie

Ratzeburger Allee 160

23538 Lübeck

E-Mail:Anna.Recke@uksh.de

\section{Zitierweise des Beitrages/Cite as:}

Sclerotherapy of spider veins

Phlebologie 2018; 47: 155-159

https://doi.org/10.12687/phleb2419-3-2018

Eingereicht: 16. März 2018

Angenommen: 16. März 2018

English version available at:

www.thieme.de/phlebo 

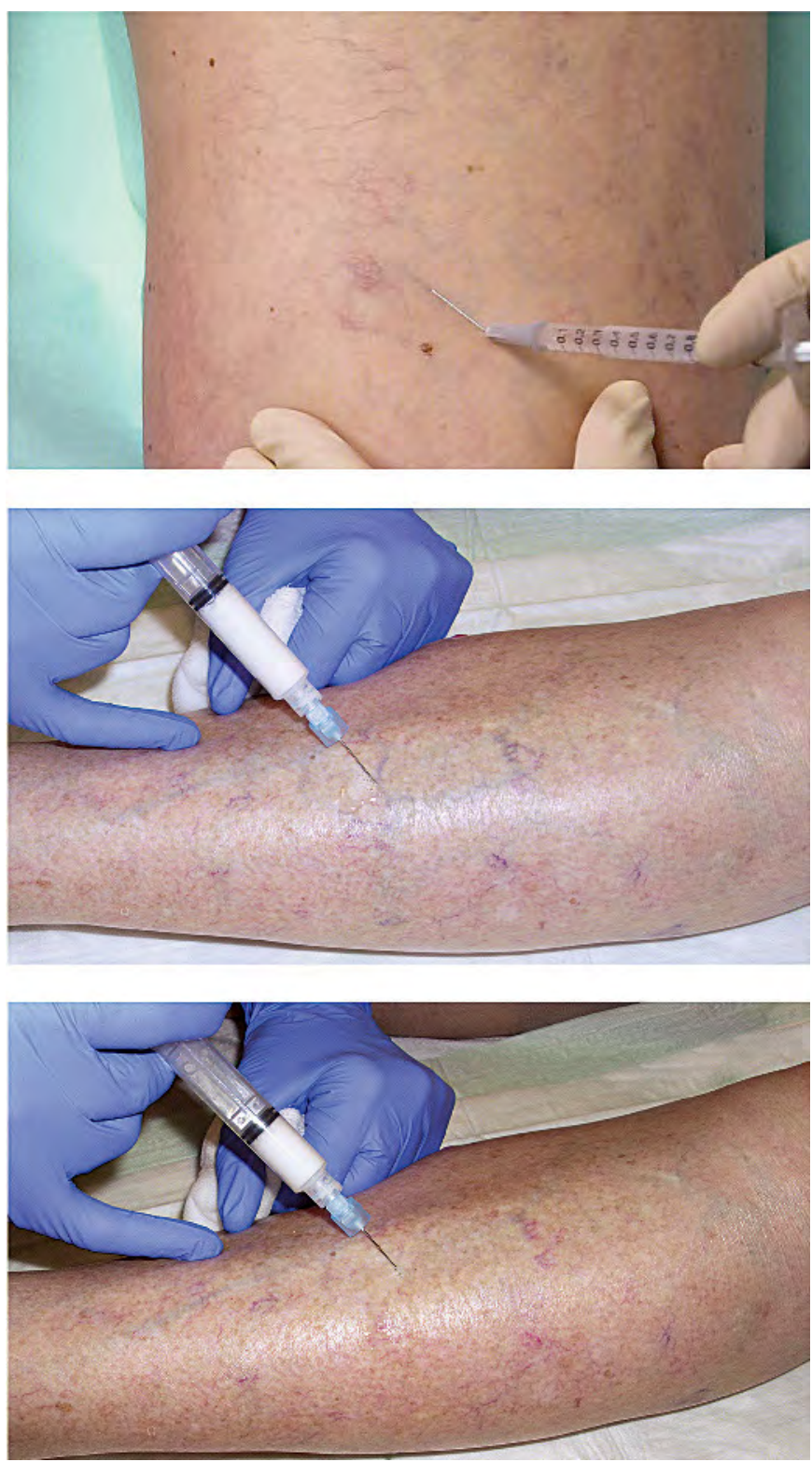

Abb. 1a

Flüssigsklerosierung von Besenreisern mit 0,5\% Polidocanol. Optimal ist die Verwendung einer $1 \mathrm{ml}$-Spritze. Pro Injektion sollten nicht mehr als 0,1-0,2 $\mathrm{ml}$ verwendet werden. Im Anschluss an die Sklerosierung erfolgt unmittelbar eine exzentrische Kompression, z.B. mit Kompressenröllchen. Über den Nutzen und die Dauer einer Kompression (Bandage/Strumpf) ist die Literatur uneinheitlich. Im Falle von Besenreisern und retikulären

Varizen führte eine Kompression über 3 Wochen zu weniger Pigmentierungen und Hämatomen und zu besserer Effektivität (vgl. 2-5).

Abb. 1b

Schaumsklerosierung von retikulären Varizen mit 0,5\% Polidocanol (1 Teil Sklerosierungsmittel +3 Teile Luft, DSS-(Doppelspritzen-)Methode). Das Gefäß kann bei sehr oberflächlicher Lage unter Sicht punktiert werden.

Abb. 1c

Gleiche Bildeinstellung wie 1B, die sklerosierte Varize ist nach Einspritzen des Schaumes nicht mehr sichtbar. Im Gegensatz zur Flüssigsklerosierung erfolgt das Anlegen der exzentrischen Kompression nicht sofort, sondern ca. 1 Minute nach der Schaumsklerosierung. 
Abb. 2a

Sklerothrombus und Hyperpigmentierung bei Z.n. Flüssigsklerosierung 2 Wochen zuvor.

Abb. 2b

Stichinzision des Sklerothrombus mittels Kanüle zur Verminderung von Hyperpigmentierungen.

Abb. 2c

Manuelle Thrombusexpression.

Abb. 2d

Exprimiertes Thrombusmaterial. Im Anschluss kann eine erneute exzentrische Kompression für ca. 12-24 Stunden erfolgen. Das Tragen eines Kompressionsstrumpfes für ca. 3 Wochen wurde empfohlen (vgl. 2-5).
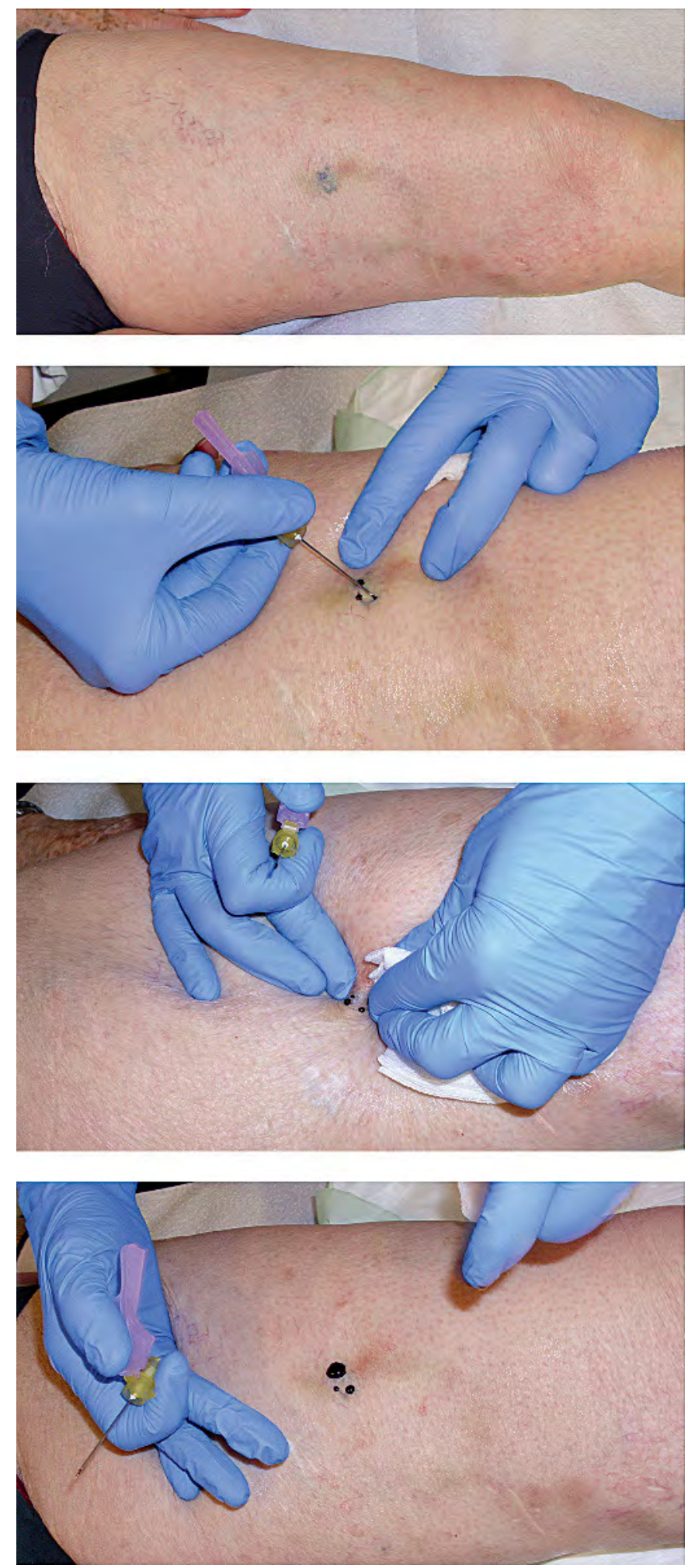


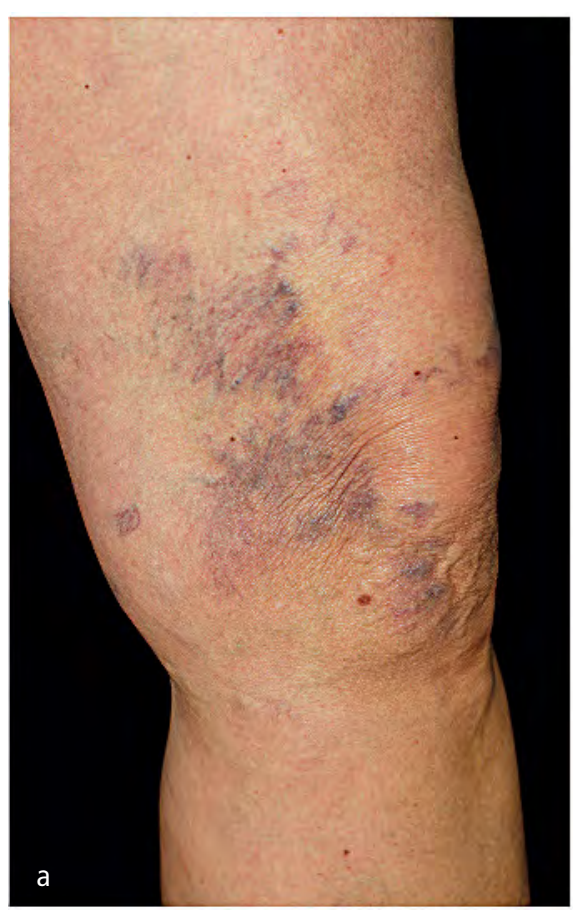

Abb. 4a Eine unerwünschte Wirkung nach Sklerosierungstherapie, aber auch nach operativen Eingriffen, ist die Neuentstehung von meist sehr feinen Teleangiektasien, das sogenannte Matting.

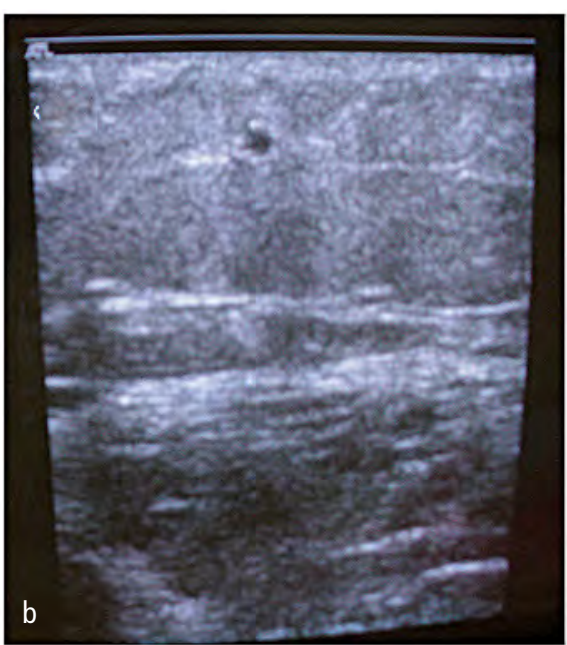

Abb. 4b Die Therapie der Wahl bei Matting ist wiederum die Sklerosierungstherapie. Hierfür ist es zielführend, sich das jeweilige Zentralgefäß des Mattings mittels Ultraschall aufzusuchen und dieses gezielt zu sklerosieren. Zur Darstellung der hier gezeigten Zentralvene wurde ein 15 MhzSchallkopf verwendet. Zur Beseitigung eines Mattings sind, je nach Ausdehnung, meistens mehrere Sklerosierungs-Sitzungen nötig.

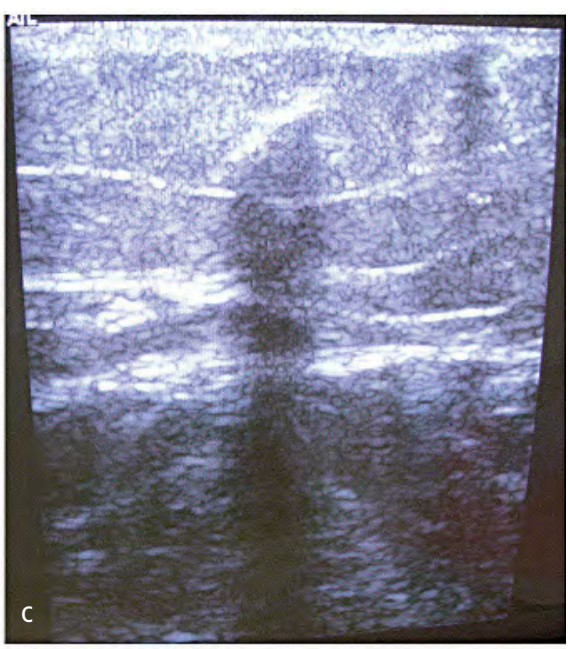

Abb. 4c Schaumsklerosierung der in Abb. 4b gezeigten Zentralvene unter sonographischer Sicht (Längsschnitt) mit 0,5\% Polidocanolschaum 1+3 nach der DSS-Methode. Die Schaumherstellung erfolgte im gezeigten Fall mit $1 \mathrm{ml}$-Spritzen, die Applikation mittels 27G-Kanüle. Der Schaum stellt sich sonographisch echoreich und mit dorsaler Schallauslöschung dar. 